\title{
Care of abdominal skin in ankylosing spondylitis patients undergoing corrective spinal surgery
}

\author{
XIAOJING SU, ZHENG WANG, BING WU, KAI SONG, YIXIU CHEN, \\ CHUNZHENG ZHANG, CHUANJUAN XUE, LILI GE and YANG LIU
}

\author{
Department of Orthopedics, General Hospital of The People's Liberation Army, Beijing 100853, P.R. China
}

Received November 12, 2018; Accepted December 15, 2020

DOI: 10.3892/etm.2021.10785

\begin{abstract}
The present study aimed to investigate the effect of a new method of abdominal skin care on patients with ankylosing spondylitis (AS) undergoing orthopedic surgery. A total of 90 patients with AS and thoracolumbar kyphosis undergoing orthopedic surgery were randomly divided into two groups. Patients in the control group received routine care while those in experimental group received the following new treatment regimen: i) Vaseline cream was applied to the abdominal skin for 3-5 min every $6 \mathrm{~h}$; ii) preoperative stretch exercises were encouraged and iii) $24 \mathrm{~h}$ after surgery, the patient was placed in a lateral decubitus position. The difference in abdominal pain levels and the size of tension blisters was the compared between the two groups. There was no difference in age, gender, disease course and kyphosis angle between two groups $(\mathrm{P}>0.05)$. Compared with the patients in the control group, those in test group experienced milder pain when evaluated by visual analogue score at 6,18 and $30 \mathrm{~h}$ after surgery $(3.6 \pm 1.2$ vs. $4.5 \pm 1.8 ; 4.4 \pm 2.3$ vs. $6.1 \pm 2.7 ; 4.1 \pm 2.1$ vs $4.1 \pm 2.1, \mathrm{P}<0.05)$. Moreover, tension vesicles in the abdominal skin were significantly smaller in the treatment group than the control group $(\mathrm{P}<0.05)$. Optimal nursing may be the key to the reduction of the level of pain and occurrence of tension vacuoles in the abdominal skin.
\end{abstract}

\section{Introduction}

Ankylosing spondylitis (AS) is an unexplained systemic disease characterized by the chronic inflammatory response of the central axis $(1,2)$. The typical age of disease onset is $16-40$ years old and the condition is more common in men (3). The main clinical manifestations of AS are low back stiffness or pain and spinal lesions, which become progressively aggravated (4), and

Correspondence to: Dr Zheng Wang, Department of Orthopedics, General Hospital of The People's Liberation Army, 28 Fuxing Road, Haidian, Beijing 100853, P.R. China

E-mail: zwang18@aliyun.com

Key words: ankylosing spondylitis, tension vacuole, skin nursing, pain spinal rigidity, deformity and dysfunction that occurs in the advanced stages of the disease and seriously affects the quality of life of patients. Patients with advanced AS are unable to walk upright, lie flat or look straight ahead and the condition may additionally affect the heart, lung and digestive function (5). Patients with AS often suffer from mental illness due to bad physical symptoms. Spinal orthopedic surgery is required to correct the long-term torso flexion deformity of patients. Following orthopedic surgery, the abdominal skin is severely stretched, often causing abdominal skin pain and tension blisters and resulting in increased patient suffering (6-9). Though surgeons pay attention to whether the patient has neurological symptoms associated with the lower limbs and whether the patient's orthosis is satisfactory, there is often less concern over the effects seen in the skin. Abdominal skin problems often seriously affect the quality of life of patients during the perioperative period. To the best of our knowledge, there are no reported solutions related to this issue. To solve this problem, the present study adopted new nursing measures for AS patients undergoing orthopedic surgery in our department and a randomized control study in comparison with traditional nursing measures was conducted.

\section{Materials and methods}

Research objective. The present study was approved by the Ethics Committee of the General Hospital, of the People's Liberation Army. A total of 90 patients with AS who had kyphosis following posterior vertebral kyphosis between January 2012 and December 2014 were selected. Patients provided their written informed consent for participation in the study and the use of their clinical data. Patients were assessed based on the Assessment of SpondyloArthritis International Society (ASAS) classification criteria (10). This study was a prospective study and sample collection was continuous. Patients were randomized into one of two groups, the experimental group or the control group. The preoperative heart and lung functions of all patients were assessed using the MAX-ii cardiopulmonary function evaluation system, where they were deemed within the normal range, and postoperative patients were awake and displayed normal vital signs (data not shown). All anesthesia was performed by single-lumen endotracheal intubation with Remifentanil. All patients were treated with an analgesic pump of the same adjusted dose and time (Remifentanil, $1 \mu \mathrm{g} / \mathrm{kg}$ ). 
Inclusion criteria. In accordance with the assessment of SpondyloArthritis international Society (ASAS) classification criteria revised in the 1984 New York Conference (11) patients displayed symptoms of cervical or cervicothoracic kyphosis; preoperative thoracic or thoracolumbar kyphosis and posterior spinal pedicle osteotomy orthopaedics (12).

Exclusion criteria. Patients with advanced AS have lower limb paralysis, heart, brain and kidney and severe hematopoietic system; high fever, tuberculosis, skin ulceration, and infectious diseases (13). Patients who did not follow the protocols were also excluded.

\section{Preoperative care}

Skin care. Skin care was performed seven days before operation, lasting for seven days. Patients in the control group were given routine preoperative guidance as follows. The abdominal skin folds were cleaned and kept dry daily and the patient was bathed in soapy water one night before surgery. After the patients in the experimental group were admitted to the hospital, the abdominal skin was cleaned daily, and vaseline cream (Guangzhou Xindi Chemical Co., Ltd.; $2 \mathrm{~g} / \mathrm{cm}^{2}$ ) was evenly applied to the abdominal skin every $3 \mathrm{~h}$ for 3-5 $\mathrm{min}$.

Abdominal skin stretching exercises. Abdominal skin stretching exercises were performed seven days before the operation, lasting for seven days. The patients in the experimental group performed daily abdominal skin stretching exercises. They were instructed to face the wall with both hands touching the wall and reach upwards, three times per day for 15-20 min each time, every 10 times for a group, complete 10 groups daily. Flexion and extension of the hip joint were in the maximum range of activity.

Psychological care. Both groups of patients were counseled preoperatively, following a course of kyphosis for one month. Corrective spinal surgery carries high risks, such as paralysis and hemorrhagic shock (14). Thus, the patients may be burdened with high psychological stress. Counseling included discussion of post-operative abdominal skin tension pain and possible tension blisters and worries about the surgery-related complications.

Intraoperative care. All patients with ankylosing spondylitis were placed in the same position during surgery, as previously described (12).

\section{Postoperative care}

Skin care. The abdominal skin was kept dry in the control group. Vaseline was smeared on the abdominal skin every $6 \mathrm{~h}$ for 3-5 min in the experimental group (Guangzhou Xindi Chemical Co., Ltd.; concentration of Vaseline cream was $\left.2 \mathrm{~g} / \mathrm{cm}^{2}\right)$.

Postoperative position. In the control group, the patients were placed in a supine position. For patients with cervical or hip ankylosis, a soft pillow was placed under the occipital and knee joints and dressing protection was applied to the appendix. In the experimental group, the left or right lateral

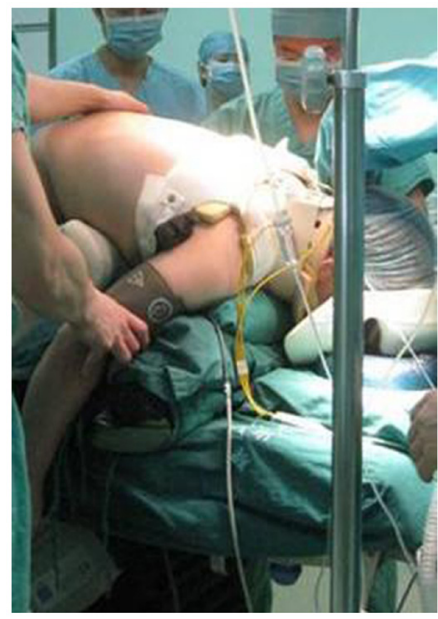

Figure 1. The process of blister care. After disinfection with aneriodine, a sterile syringe was used in the lower part of the blister to draw out the liquid, and the blister wall was gently pressed and draped on the skin to prevent further damage. A $3 \mathrm{M}$ transparent film protection was used until the blisters had completely disappeared.

decubitus position was alternated within $24 \mathrm{~h}$ after patients returned from the operating theater to the ward. Cushions were used between the knees, and under bilateral ankles to avoid decubitus ulcer.

Tension blister care. In both the control and experimental groups, once tension blisters appeared, they were carefully protected using the $3 \mathrm{M}$ transparent film. After 1-2 days, the swelling of the affected limb may have been reduced and the blisters may have formed a scar. If the blisters were large (over $2 \mathrm{~cm}$ in diameter) (15), after disinfection with aneriodine, a sterile syringe was used in the lower part of the blister to draw out the liquid and the blister wall gently pressed and draped on the skin to prevent further damage. A 3M transparent film protection was used until the blisters had completely disappeared (Fig. 1).

Visual analogue score (VAS). At 1 day before surgery and 6 day after surgery, the occurrence of tension vesicles was observed and recorded every $12 \mathrm{~h}$, and the maximum diameter of the vesicles was graded. The VAS of the abdominal skin pain was recorded. The method involves a rating on a 10 point scale with 0 being no pain and 10 being unbearable severe pain (16). The scoring criteria were as follows: 0 , no pain; $0<-3$, slight pain that could be endured; $4-6$, the patient's pain affected sleep quality but was tolerable; 7-10, the patient had progressively strong pain up to a level that was unbearable, affecting appetite and sleep. Each measurement was assessed by two experienced nurses to avoid subjective errors.

Statistical methods. All analyses were performed using SPSS computer software (version 13; SPSS, Inc.). An independent sample t-test was used for the two groups of measurement data. The chi-square test was used to compare the count data. The occurrence of tension blisters was compared by the one-way sequence Wilcoxon rank sum test. $\mathrm{P}<0.05$ was considered to be statistically significant. 
Table I. Comparison of general conditions between the two groups of patients.

\begin{tabular}{lcccc}
\hline Variable & Test group $(\mathrm{n}=45)$ & Control group $(\mathrm{n}=45)$ & $\chi^{2} / \mathrm{t}$ & P-value \\
\hline Age (years) & $40.3 \pm 9.3$ & $38.9 \pm 9.4$ & 0.710 & 0.479 \\
Sex (male) & 37 & 40 & $0.809^{\mathrm{a}}$ & 0.368 \\
Front and rear lobe $\left(^{\circ}\right)$ & $73.7 \pm 17.8$ & $76.5 \pm 21.1$ & 0.680 & 0.498 \\
Weight (kg) & $62.4 \pm 13.1$ & $58.9 \pm 10.2$ & 1.414 & 0.161 \\
Course of the disease (years) & $14.2 \pm 7.6$ & $11.0 \pm 8.2$ & 1.920 & 0.058 \\
\hline
\end{tabular}

${ }^{\mathrm{a}} \chi^{2}$, other values are $\mathrm{t}$ values.
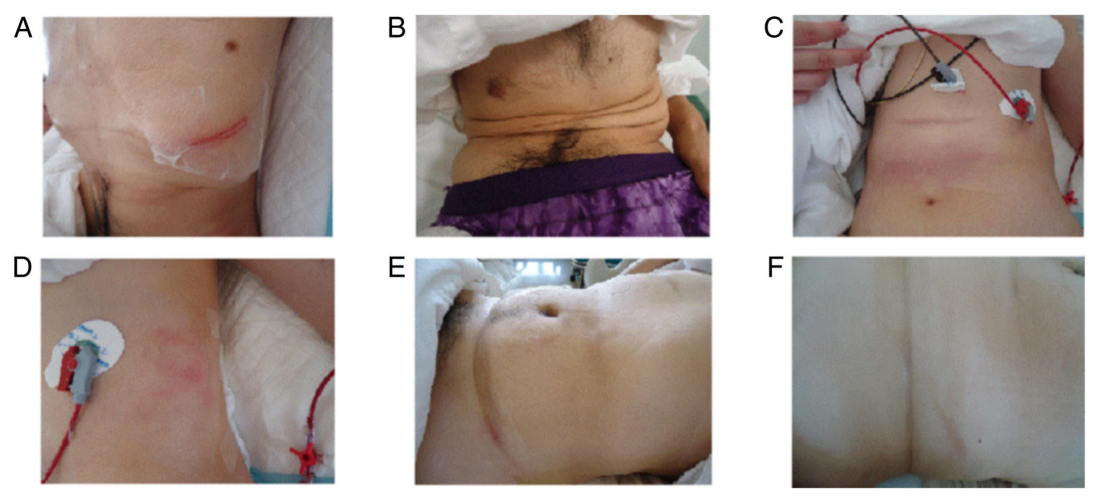

Figure 2. The conditions of different skins from postoperative 1 day to postoperative 6 day. (A) Damaged skin. (B) Concave skin. (C) Skin with tension blisters (D) Damaged skin. (E) Damaged skin. (F) Concave skin.

\section{Results}

Comparison of demographics between the two groups of patients. A randomized digital table method was used to divide the selected patients into two groups. There were 45 patients in the experimental group, 37 males and 8 females, with a mean age of (40.3 \pm 9.3$)$ years (25-67 years). There were a further 45 patients in the control group, 40 males and 5 females, with a mean age of (38.9 \pm 9.4$)$ years (23-70 years). There was no significant difference in the age at surgery, sex, duration of disease, body weight or anterior and posterior convex angle between the experimental group and the control group ( $\mathrm{P}>0.05$; Table I and Fig. 2). The average range of spinal deformity in the experimental group before surgery was $58.4 \pm 16.1^{\circ}$, and postoperatively was $-2.8 \pm 15.0^{\circ}$. The average range of spinal deformity in the control group before surgery was $56.7 \pm 18.3^{\circ}$, and postoperatively was $-8.3 \pm 16.6^{\circ}$.

Comparison of abdominal pain scores between the groups. At 6, 18 and $30 \mathrm{~h}$ after surgery, the abdominal pain scores of the experimental group were significantly lower than those of the control group $(\mathrm{P}<0.05)$. There was no significant difference in pain levels between the two groups after $42 \mathrm{~h}(\mathrm{P}>0.05)$, and the pain returned to the preoperative level at $66 \mathrm{~h}$ after surgery (Fig. 3).

Comparison of abdominal tension vesicles in two groups. All vesicles from all patients were detected. Tension vesicles in the abdominal skin of the experimental group were significantly smaller in the treatment group than in the control group, and

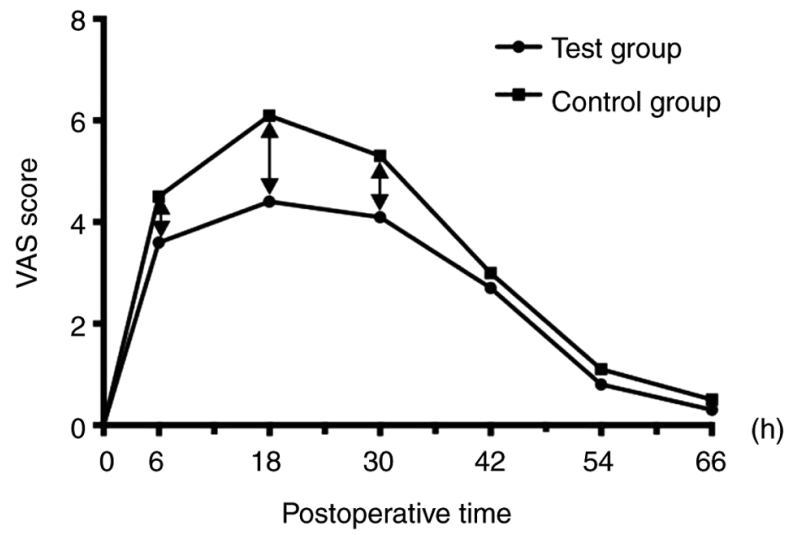

Figure 3. Changes in VAS scores of abdominal skin within $66 \mathrm{~h}$ after surgery in both groups. The VAS score was determined $1 \mathrm{~h}$ before surgery. The double arrow indicates time points at which the VAS score was statistically significant between the two groups $(\mathrm{P}<0.05) .45$ patients in each group. All patients measured at all-time points. one-way ANOVA was used to perform comparison. VAS, visual analogue score.

the difference was statistically significant $(\mathrm{u}=2.238>1.96$, $\mathrm{P}<0.05$; Table II).

\section{Discussion}

The development of tension blisters is often caused by excessive swelling of the skin, excessive pressure and blood circulation disorders. When venous return is blocked, local venous congestion and increased permeability of the blood 
Table II. Comparison of tension blister size.

\begin{tabular}{lccr}
\hline Size & $\begin{array}{c}\text { Total number of } \\
\text { blisters; Test group }(\mathrm{n}=45)\end{array}$ & $\begin{array}{c}\text { Total number of } \\
\text { blisters; Control group }(\mathrm{n}=45)\end{array}$ & P-value \\
\hline $0-0.50 \mathrm{~cm}$ & 22 & 15 & $<0.05$ \\
$0.51-1.00 \mathrm{~cm}$ & 12 & 10 & $<0.05$ \\
$1.01-1.50 \mathrm{~cm}$ & 8 & 7 & $<0.05$ \\
$1.51-2.00 \mathrm{~cm}$ & 2 & 8 & $<0.05$ \\
$>2.00 \mathrm{~cm}$ & 1 & 5 & $<0.05$ \\
\hline
\end{tabular}

Wilcoxon Comparison of two samples of grade data: $\mathrm{u}=2.238>1.96, \mathrm{P}<0.05$.

vessels cause small blisters to form in the epidermis $(17,18)$. Patients with AS have long-term contracture deformity of the trunk before surgery $(19,20)$. Relaxation of the skin on the abdominal wall is far worse than that of normal people. After orthopedic surgery, the skin of the abdomen will be distended, causing high tension of the skin and subcutaneous blood circulation dysfunction, pain and tendency to develop tension blisters $(21,22)$. Unnecessary trouble and pain impact postoperative prognosis and psychology (23). Therefore, reducing the occurrence of tension blisters is of great significance for clinical nursing work.

Patients undergoing general anesthesia for spinal surgery are usually placed in a conventional position in which the pillow is placed flat and the head is biased to one side, or the lower neck and the lower occipital pillow are raised to a head position of $10-15^{\circ}$. Due to the unstable vital signs of early patients, the bed was raised to a high angle to prevent hemodynamic changes and fluctuations in cardiopulmonary function. The modified semi-recumbent position can only raise the head by $10-15^{\circ}$. The purpose of the supine position is to prevent aspiration pneumonia caused by vomiting when the patient is not awake (24). The main advantage of the improved semi-recumbent position is that the patient's comfort can be improved to some extent. However, patients with AS kyphosis are different from patients with general scoliosis or other spinal diseases in a number of ways (25): i) Patients with AS have different degrees of inflammation or ankylosing damage in the cervical spine before surgery, several patients have cervical vertebrae or even full rigidity, and when they are supine, they cannot move to lie on their side; ii) as patients with AS kyphosis have difficulties entering the supine position before surgery, they are used to alternating between the left and right lateral positions in bed. If the patient is supine after surgery, psychological and physical discomfort will occur; iii) even after kyphotic orthopedic surgery, the sagittal balance of patients with AS kyphosis does not necessarily reach the normal spine-pelvic parameter standard and a kyphosis angle greater than the normal value remains. The comfort level of the supine position is significantly lower than that of the lateral position; iv) some patients have hip flexion deformity before the operation. In the supine position after spinal orthopedic surgery, the bilateral hip and knee joints should be flexed. Even if the knee joint is placed under the cushion, the lower limb muscle tone and hip pain of the patient can be increased compared with the lateral position.
Postoperative exercise can maintain the normal range of motion of the thorax and the normal shape and flexibility of the spine. In addition, postoperative exercise can maintain the motor function of limbs, prevent or reduce limb muscle atrophy, increase bone mineral density and prevent osteoporosis.

AS patients usually experience a reduction in pain at the incision within 7 days after surgery (26). The results of the present study confirmed that the abdominal wall tension pain and back incision pain were significantly lower in the treatment group than in the control group. One possible for reason for this may be the lateral position of the patient in the treatment group. The patient flexes the lower limbs when lying in a lateral position, which reduces the sharp increase of the abdominal wall tension caused by the kyphosis. Additionally, a lateral position reduces the pressure on the back incision. An increase in abdominal wall tension is related to the formation of tension vesicles in the abdominal wall of the patient, therefore a sharp increase in the abdominal wall tension caused by kyphosis may also reduce the formation of tension vesicles. Patients with AS have long-term contracture deformity of the trunk before surgery. The relaxation of the skin on the abdominal wall is worse than that of normal people. After orthopedic surgery, the skin of the abdomen will be pulled tight, causing a high degree of tension in the skin, which increases pain and causes the development of tension blisters. Flexing the torso and the hips and knees through the lateral position can reduce the tension of the skin to a certain extent, thereby reducing pain and reducing the occurrence of tension blisters. In the lateral position, reasonable care measures were taken, and there was no significant difference in the incidence of stage I pressure ulcers between the two groups. In addition, there was no significant effect on the pain of the patient's back incision. Overall, the lateral position reduced abdominal discomfort without increasing the patient's anesthesia complications and the patient's back incision pain. AS patients undergoing orthopedic surgery in a lateral position had the following advantages: i) The patients bed habits could be monitored to improve their comfort before the start of the operation; ii) a reduction in abdominal wall tension reduced abdominal wall pain and abdominal wall tension blisters occurrence; iii) reduced pressure on the back incision reduced the pain of the incision and iv) the occurrence of vomiting and aspiration in patients was avoided. The present study showed that patients with AS kyphosis with the experimental treatment did not experience increased respiratory rate changes and a low incidence of postoperative adverse 
events such as vomiting, dizziness and stage I pressure sores after orthopedic surgery. On the contrary, the experimental treatment also relieved the patient's abdominal wall pain and back incision pain and reduced the patient's heart rate and the incidence of abdominal wall tension blisters, which is conducive to increasing patient comfort, reducing patient suffering and promoting body recovery.

Vaseline is an oily substance that forms a protective film on the skin to prevent evaporation of water, softening of the skin (27). Vaseline also reduces friction and shear on the skin (28). Preoperative abdominal skin stretching training also plays a role in relaxing the skin to a certain extent (29-31). In addition, the left and right lateral decubitus position of the postoperative patient put the torso, hips and knees in a slightly flexed position, which can reduce the tension of the skin to a certain extent, thereby reducing pain and reducing the occurrence of tension blisters (32).

The results of the present study suggested that patients with AS kyphosis experienced reduced pain and tension of the abdominal skin after surgery by combined use of Vaseline cream smear, preoperative abdominal skin stretching training and postoperative alternative lateral decubitus positions change in comparison with patients that received standard treatment. Also, the occurrence of was blisters reduced. Optimal nursing is the key to ease the pain and tension vacuole in abdominal skin.

\section{Acknowledgements}

Not applicable.

\section{Funding}

No funding was received.

\section{Availability of data and materials}

The datasets used and/or analyzed during the current study are available from the corresponding author on reasonable request.

\section{Authors' contributions}

XS and BW were responsible for conception and design. ZW, LG and YC carried out analysis and interpretation of data. KS, YL, CZ and CX were responsible for acquisition of data. All authors have read and approved this final version of this article for publication. The authenticity of all the raw data have been assessed by XS and ZW.

\section{Ethics approval and consent to participate}

Patients provided their written informed consent for participation in the study and the study received ethical approval from the Ethics Committee of the General Hospital of the People's Liberation Army.

\section{Patient consent for publication}

The patents provided their written informed consent for the publication of their data.

\section{Competing interests}

The authors declare that they have no competing interests.

\section{References}

1. Thakkar SJ, Grayburn PA, Hall SA and Roberts WC: Orthotopic heart transplantation for ankylosing spondylitis masquerading as nonischemic cardiomyopathy. Am J Cardiol 123: 1732-1735, 2019.

2. Nunes GPS, Cunha PDS, Bosco DPD and Ribeiro SLE: Challenging management of hepatitis B infection in ankylosing spondylitis patients in an endemic area during immunosuppressive therapy. Rev Soc Bras Med Trop 52: e20180386, 2019.

3. Moura CS, Rahme E, Maksymowych WP, Abrahamowicz M, Bessette L and Bernatsky S: Use of disease-modifying anti-rheumatic or anti-tumour necrosis factor drugs and risk of hospitalized infection in ankylosing spondylitis. Scand J Rheumatol 48: 121-127, 2019.

4. Qian Q, Xu X, He H, Ji H, Zhang H, Ding Y, Dai SM, Zou Y, Zhu Q, Yang C, et al: Clinical patterns and characteristics of ankylosing spondylitis in China. Clin Rheumatol 36: 1561-1568, 2017.

5. Golder V and Schachna L: Ankylosing spondylitis: An update. Aust Fam Physician 42: 780-784, 2013.

6. Dębska J, Kotulska-Józwiak K, Pawliszak P, Bilska M, Chmielewski D, Puzio I and Jurkiewicz E: Spinal cord lesions in children and adolescents with multiple sclerosis-Magnetic resonance imaging. Neurol Neurochir Pol 51: 77-81, 2017.

7. Braun J and Sieper J: Ankylosing spondylitis. Lancet 369: 1379-1390, 2007.

8. Cozowicz C, Olson A, Poeran J, Mörwald EE, Zubizarreta N, Girardi FP, Hughes AP, Mazumdar M and Memtsoudis SG: Opioid prescription levels and postoperative outcomes in orthopedic surgery. Pain 158: 2422-2430, 2017.

9. Memtsoudis SG, Vougioukas VI, Stundner O and Poultsides LA: Perioperative outcomes in orthopedic surgery. ScientificWorldJournal 2015: 648284, 2015.

10. Sieper J, Rudwaleit M, Baraliakos X, Brandt J, Braun J, Burgos-Vargas R, Dougados M, Hermann KG, Landewé R, Maksymowych W and van der Heijde D: The assessment of SpondyloArthritis international society (ASAS) handbook: A guide to assess spondyloarthritis. Ann Rheum Dis 68 (Suppl 2): iil-ii44, 2009.

11. Lilje SC, Persson UB, Tangen ST, Kåsamoen S and Skillgate E: Costs and utilities of manual therapy and orthopedic standard care for low-prioritized orthopedic outpatients of working age: A cost consequence analysis. Clin J Pain 30: 730-736, 2014.

12. Feng J, Zhou J, Huang M, Xia P and Liu W: Clinical and radiological outcomes of the multilevel Ponte osteotomy with posterior selective segmental pedicle screw constructs to treat adolescent thoracic idiopathic scoliosis. J Orthop Surg Res 13: 305, 2018.

13. Dundar U, Solak O, Toktas H, Demirdal US, Subasi V, Kavuncu V and Evcik D: Effect of aquatic exercise on ankylosing spondylitis: A randomized controlled trial. Rheumatol Int 34: 1505-1511, 2014.

14. Passias PG, Poorman GW, Jalai CM, Line B, Diebo B, Park P, Hart R, Burton D, Schwab F, Lafage V, et al: Outcomes of open staged corrective surgery in the setting of adult spinal deformity. Spine J 17: 1091-1099, 2017.

15. Li D, Liu Y, Qi L, Gu J, Tang Q, Wang X and Bhushan B: Properties of blisters formed on polymer films and differentiating them from Nanobubbles/Nanodrops. Langmuir 35: 3005-3012, 2019.

16. Heller GZ, Manuguerra M and Chow R: How to analyze the visual analogue scale: Myths, truths and clinical relevance? Scand J Pain 13: 67-75, 2016.

17. Song K, Zheng G, Zhang Y, Zhang X, Mao K and Wang Y: A new method for calculating the exact angle required for spinal osteotomy. Spine (Phila Pa 1976) 38: E616-E620, 2013.

18. Malone PC and Agutter PS: To what extent might deep venous thrombosis and chronic venous insufficiency share a common etiology? Int Angiol 28: 254-268, 2009.

19. Ciciarello F, Mandolesi S, Galeandro AI, Marceca A, Rossi M, Fedele F, Gesualdo M, Cortese F, Zito A, Federico F, et al: Age-related vascular differences among patients suffering from multiple sclerosis. Curr Neurovasc Res 11: 23-30, 2014.

20. Dong FH: Precise application of Traditional Chinese medicine in minimally-invasive techniques. Zhongguo Gu Shang 31: 493-496, 2018 (In Chinese). 
21. Deslauriers N, Jacques F, Danino A, Harris P, Martin J, Liberman M, Duranceau A and Ferraro P: Lateral mediastinal tracheostomy in benign disease: An uncommon procedure for a rare indication. Ann Thorac Surg 89: 979-981, 2010.

22. Sakai T, Sawada M, Sato Y, Kimura F, Yagihashi N, Iwabuchi T, Kimura D, Tsushima T and Hatanaka R: Tension pneumothorax developing hemothorax after chest tube drainage. Kyobu Geka 69: 991-994, 2016.

23. Rutström E, Söndergaard S, Lundborg C and Ene K: Postoperative pain experience, pain treatment and recovery after lumbar fusion and fixation surgery. Int J Orthop Trauma Nurs 34: 3-8, 2019.

24. Hassan EA and Baraka AAE: The effect of reverse Trendelenburg position versus semi-recumbent position on respiratory parameters of obese critically ill patients: A randomized controlled trial. J Clin Nurs: Jan 12, 2021 doi: 10.1111/jocn.15645 (Epub ahead of print).

25. Zhang H, Zhang X, Hu F, Hu W, Wang Y and Hao Y: Ankylosing spondylitis kyphosis surgical correction postoperative evaluation via SRS-22 domain investigation. J Orthop Surg Res 13: $5,2018$.

26. Weibel S, Jelting Y, Pace NL, Helf A, Eberhart LH, Hahnenkamp K, Hollmann MW, Poepping DM, Schnabel A and Kranke P: Continuous intravenous perioperative lidocaine infusion for postoperative pain and recovery in adults. Cochrane Database Syst Rev 6: CD009642, 2018
27. Wu Y,Xu Y, Shi J and Zhu Y: Surgical treatment of Rüedi-Allgöwer III type Pilon Fractures. Zhongguo Xiu Fu Chong Jian Wai Ke Za Zhi 28: 828-831, 2014 (In Chinese).

28. Wolf L, Eertmans F, Wolf D, Rossel B and Adriaens E: Efficacy and safety of a mineral Oil-based head lice shampoo: A randomized, controlled, investigator-blinded, comparative study. PLoS One 11: e0156853, 2016.

29. Tlougan BE, Mancini AJ, Mandell JA, Cohen DE and Sanchez MR: Skin conditions in figure skaters, ice-hockey players and speed skaters: Part I-mechanical dermatoses. Sports Med 41: 709-719, 2011.

30. Srinivas A, Kaman L, Raj P, Gautam V, Dahiya D, Singh G, Singh R and Medhi B: Comparison of the efficacy of chlorhexidine gluconate versus povidone iodine as preoperative skin preparation for the prevention of surgical site infections in clean-contaminated upper abdominal surgeries. Surg Today 45: 1378-1384, 2015

31. Ingvaldsen CA, Tønseth KA, Pripp AH and Tindholdt TT: Microcirculatory evaluation of the abdominal skin in breast reconstruction with deep inferior epigastric artery perforator flap. Plast Reconstr Surg Glob Open 4: e616, 2016.

32. Ikegami S, Kamimura M, Uchiyama S, Nakamura $Y$, Mukaiyama $\mathrm{K}$ and Kato $\mathrm{H}$ : Clinical implications of hip flexion in the measurement of spinal bone mineral density. J Clin Densitom 19: 270-276, 2016. 